Administrative Überlast im steten Zunehmen begriffen lautet die Diagnose. Unsere Patientinnen und Patienten wünschen sich mehr Zuwendung und Zeit von uns Ärztinnen und Ärzten, und gleichzeitig zwingt die zunehmende Regulierungsdichte zu immer mehr Dokumentation und Administration. Bevor wir also nur noch Röntgenbilder und Laborwerte behandeln, und der Frustrationspegel überall steigt, sind Massnahmen dringend nötig. Dr. Volker Schade stellt hier ein Instrument für die administrative Entlastung der Ärzte und eine sinnvolle Aufgabenverteilung vor. Wesentlich sind auch hier individuelle Analysen und persönliche Beratungen, welche die konkrete Problematik angehen.

Dr. med. Christoph Bosshard, Vizepräsident der FMH, Departementsverantwortlicher DDQ/SAQM

\title{
Zum Kern ärztlichen Tuns
}

\section{Volker Schade}

Dr., Centrum für PersonalManagement und Organisationsgestaltung Bern (cpmo)

Die administrative Belastung der Spitalärztinnen und -ärzte (nachfolgend: Ärzte) ist hoch. Diese Zeit geht für die direkte Patientenbetreuung verloren. Die aktuelle Begleitstudie im Auftrag der FMH zu den Auswirkungen der neuen Spitalfinanzierung ergab, dass der administrative Aufwand für die Ärzte weiter steigt. Die SÄZ berichtete darüber. Ohne Zweifel ist das Thema hochaktuell. Zudem scheint der Trend des zunehmenden administrativen Aufwands der Ärzte in den letzten Jahren stabil zu sein. Eine Umkehr ist nicht in Sicht. Es braucht also kreative Ideen, wie mit dieser Problematik künftig umgegangen werden kann. Ein mehrfach erprobter Lösungsansatz wird nachfolgend beschrieben.

\section{Noch mehr Administration und kein Ende?}

Die hohe zeitliche und inhaltliche Belastung der Ärzte in den Spitälern ist Realität. Ärzte haben trotz gesetzlicher Regelungen immer noch eine zu hohe Anzahl an Überstunden. Die Mitgliederbefragung des VSAO 2014 zur Arbeitsbelastung der Assistenz- und Oberärztinnen und -ärzte zeigte, dass die durchschnittliche wöchentliche Arbeitszeit 6,5 Stunden über der gesetzlich festgelegten Höchstarbeitszeit von 50 Stunden lag [1]. Jeder vierte Assistenz- oder Oberarzt arbeitete sogar im Durchschnitt mehr als 60 Stunden pro Woche. Nur 29\% der an der Befragung teilnehmenden Ärzte konnten die im Arbeitsvertrag festgelegte Wochenarbeitszeit einhalten [2]. Das sind alarmierende Zeichen, aus arbeitsgesetzlicher Sicht und aus einer Belastungsperspektive ohnehin. Der VSAO empfiehlt u.a. eine Entlastung der Ärzte von administrativen Aufgaben [3].
Studien zeigen, dass administrative Aufgaben einen (zu) grossen Teil der Arbeit der Ärzte ausmachen. In der oben erwähnten Begleitstudie SwissDRG wurde der Zeitaufwand der Ärzte in der Akutsomatik analysiert. Der Anteil an patientennahen Tätigkeiten umfasst gerade noch rund ein Drittel der ärztlichen Arbeitszeit [4]. Und dieser Anteil hat seit 2011 deutlich abgenommen. Als unglücklicher Gegentrend hat die administrative Belastung im Vergleichszeitraum zugenommen. Das ist Zeit, die dann für wichtige ärztliche Kerntätigkeiten fehlt. Auch die NZZ nahm sich jüngst des Themas an: «Mediziner versinken in der Papierflut», titelte sie [5]. Besonders betroffen sind die Assistenzärzte. Was sind die Konsequenzen der hohen Belastung?

\section{Konsequenzen der Überlastung}

Unter der zu hohen Belastung leidet sowohl die psychische wie auch die physische Gesundheit der Ärzte. 
In der aktuellen Begleitstudie SwissDRG beurteilten 11\% der Spitalärzte die eigene physische Gesundheit als gerade ausreichend. 3\% der Ärzte gaben an, häufig oder meistens an einer Depression zu leiden, und rund 80\% der Ärzte sind nach eigenen Angaben andauerndem Zeit- und Leistungsdruck ausgesetzt. Ein unzureichendes physisches und psychisches Wohlbefinden der Ärzte hat für den Patienten schwerwiegende Konsequenzen, da in diesem Fall die Versorgungsqualität abnimmt. Dies bestätigen auch die Ärzte selbst. Nur gerade ein Fünftel der Ärzte glaubt, dass die Qualität der Patientenversorgung selten oder nie durch die hohe Arbeitsbelastung oder den Zeitdruck beeinträchtigt wird [6]. Die Wahrscheinlichkeit von patientenbezogenen Komplikationen erhöht sich. 38\% der befragten Ärzte haben mindestens einmal in den letzten zwei Jahren eine Gefährdung von Patienten durch berufliche Übermüdung miterlebt [7].

Auch die Weiterbildung der Assistenzärzte leidet unter der hohen Belastung. Nur gerade jeder zweite Assistenzarzt kann seine Weiterbildung während der Arbeitszeit zu seiner vollen Zufriedenheit erfüllen [8]. Der Ärztemangel verstärkt die ganze Problematik zusätzlich.

\section{Illegitime Aufgaben}

Welche Auswirkungen kann das beschriebene Ungleichgewicht von ärztlichen Kernaufgaben und «Nebentätigkeiten» haben? Besonders administrative Aufgaben werden von den Ärzten häufig als «illegitim» erlebt. Darunter sind Tätigkeiten zu verstehen, die für die eigene Funktion als unzumutbar empfunden werden oder gar unnötig sind. Illegitime Aufgaben respektieren die berufliche Identität nicht, sondern erfordern, dass man Aktivitäten ausführen muss, die dem Kern der beruflichen Rolle nicht entsprechen. Dies führt zu einer Gefährdung des Selbstwerts. Das gilt als Stressfaktor.

Es konnten Zusammenhänge zwischen illegitimen Aufgaben und Indikatoren psychischer Gesundheit gefunden werden. Ein hohes Ausmass an illegitimen Aufgaben führt zu negativen Gefühlen gegenüber der eigenen Organisation, psychosomatischen Beschwerden und einer geringen Arbeitszufriedenheit [9]. Es scheint auf der Hand zu liegen: Der hohe Anteil der administrativen Aufgaben der Ärzte und deren weitere Zunahme stellen ein seriöses Problem dar, welches eine Reihe von Folgeproblemen verursacht. Nicht zuletzt ist die logische Konsequenz auch ein Anstieg der Kosten für die Spitäler. Ansätze sind also gefragt, die zu einer Reduktion des administrativen Aufwands der Ärzte führen. Das dürfte sich kostenschonend auswirken.

\section{Ein Lösungsansatz: Hospitalisationsmanagement}

Administration in Spitälern ist nicht an sich zu verdammen. Sie hilft, der Organisation Ordnung und Gewissheit zu geben. Sie kann sich aber auch gegen die Interessen des Unternehmens und der darin Tätigen richten. Das tritt z.B. ein, wenn Ärzte immer mehr Aufgaben erledigen müssen, die nicht im Kern der ärztlichen Tätigkeit liegen. Ein solches Szenario stand sicher nicht im Zentrum der Berufswahl der allermeisten Ärzte. Im Kern «nicht-ärztliche» Aufgaben sollten demnach, soweit möglich, dafür ausgebildeten Mitarbeitenden übertragen werden.

Anlass für unsere intensive Beschäftigung mit dieser Thematik waren Bemühungen um die Reduktion der ärztlichen Arbeitszeiten, die mit arbeitszeitlichen Regelungen nicht mehr vereinbar waren. Das Inselspital Bern hat hier in den frühen 2000er Jahren eine Vorreiterrolle eingenommen. Dort wurde in Zusammenarbeit von internen und externen Spezialisten ein Konzept entwickelt, welches die Ärzte zielgerichtet entlastet. Administrative Tätigkeiten, die zeitraubend sind, wurden von den Ärzten auf eine administrativ tätige Person übertragen. Dieses Vorgehen war so erfolgreich, dass wir es fortan laufend weiterentwickelt haben.

Das «Hospitalisationsmanagement» war geboren. Die Kernaufgabe des Hospitalisationsmanagers bwz. der Hospitalisationsmanagerin (nachfolgend: Hospitalisationsmanager) besteht darin, sich vor, während und nach dem Spitalaufenthalt um die Patientenakten sowie um die Organisation notwendiger Aktivitäten rund um den Patienten zu kümmern (siehe Tabelle 1). Die Ärzte werden dadurch entlastet und gewinnen Zeit. Zudem stellt der Hospitalisationsmanager eine Ansprechperson für interne und externe Stellen dar.

Tabelle 1: Hauptaufgaben des Hospitalisationsmanagers.

Eintrittsvorbereitung, z.B.

- Eintrittsunterlagen des Patienten zusammenstellen

- Spitaleintritt vorbereiten

(«Hintergrund»-)Aufgaben um den Patienten herum

während des stationären Aufenthalts, z.B.

- Patientendossier vervollständigen

- Berichte, Befunde anfordern

- Untersuchungen anmelden, Konsilien vereinbaren

Austrittsvorbereitung, z.B.

- Administrative Abwicklung des Spitalaustritts

mit den involvierten Stellen koordinieren

- Organisation Reha 


\section{Implementierung des Hospitalisations- managements}

In unserer arbeitswissenschaftlichen Tätigkeit in Schweizer Spitälern waren wir in den vergangenen 15 Jahren zunehmend mit der Thematik konfrontiert, wie die administrative Belastung der Ärzte reduziert werden kann. Im Zuge der Umstrukturierung der Aufgabenverteilung wurde das Hospitalisationsmanagement in verschiedenen Kliniken und Spitälern erfolgreich eingeführt. Abbildung 1 zeigt, wie der Ablauf eines solchen Hospitalisationsmanagement-Projekts aussieht.

In einem ersten Schritt wird die spezifische Situation der Klinik / des Spitals mittels Fragebogen, Interviews sowie Tätigkeitsbeobachtungen erhoben. Auf dieser Basis werden wünschenswerte Soll-Zustände für die jeweilige Klinik erarbeitet. Es werden konkrete Entlastungsmöglichkeiten für die Ärzte vorgeschlagen und das Aufgabenportfolio des Hospitalisationsmanagers erstellt. Anschliessend wird ein Hospitalisationsmanager rekrutiert und in seine Aufgaben eingeführt. So viel in aller Kürze zum Ablauf. Was bringt das nun?

\section{Hospitalisationsmanagement: Administration der Ärzte verringert!}

Aus der Evaluation bisher umgesetzter Projekte resultieren vielversprechende Ergebnisse. In einer Klinik

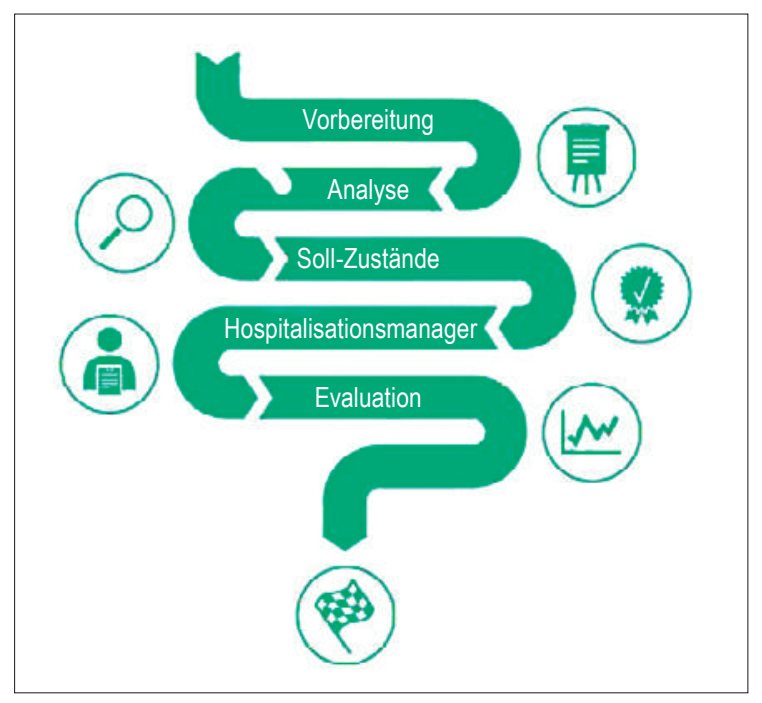

Abbildung 1: Ablauf zur Implementierung des Hospitalisationsmanagements (Grafik: zVg vom Autor).

konnte die Arbeitszeit pro Tag und Assistenzarzt um ca. 35 Minuten reduziert werden, dies trotz gleichzeitig gestiegenem Arbeitsvolumen [10]. Der Anteil an administrativen Aufgaben für die Assistenzärzte wurde in allen evaluierten Projekten durch das Hospitalisationsmanagement verringert, z.T. bis zu $50 \%$. Dadurch stand deutlich mehr Zeit für ärztliche Kerntätigkeiten, wie die Arbeit am Patienten, Weiterbildung oder Forschung, zur Verfügung. Klarere Zuständigkeiten, effizienteres Arbeiten sowie eine geringere Fragmentierung der ärztlichen Tätigkeit zählen zu den weiteren

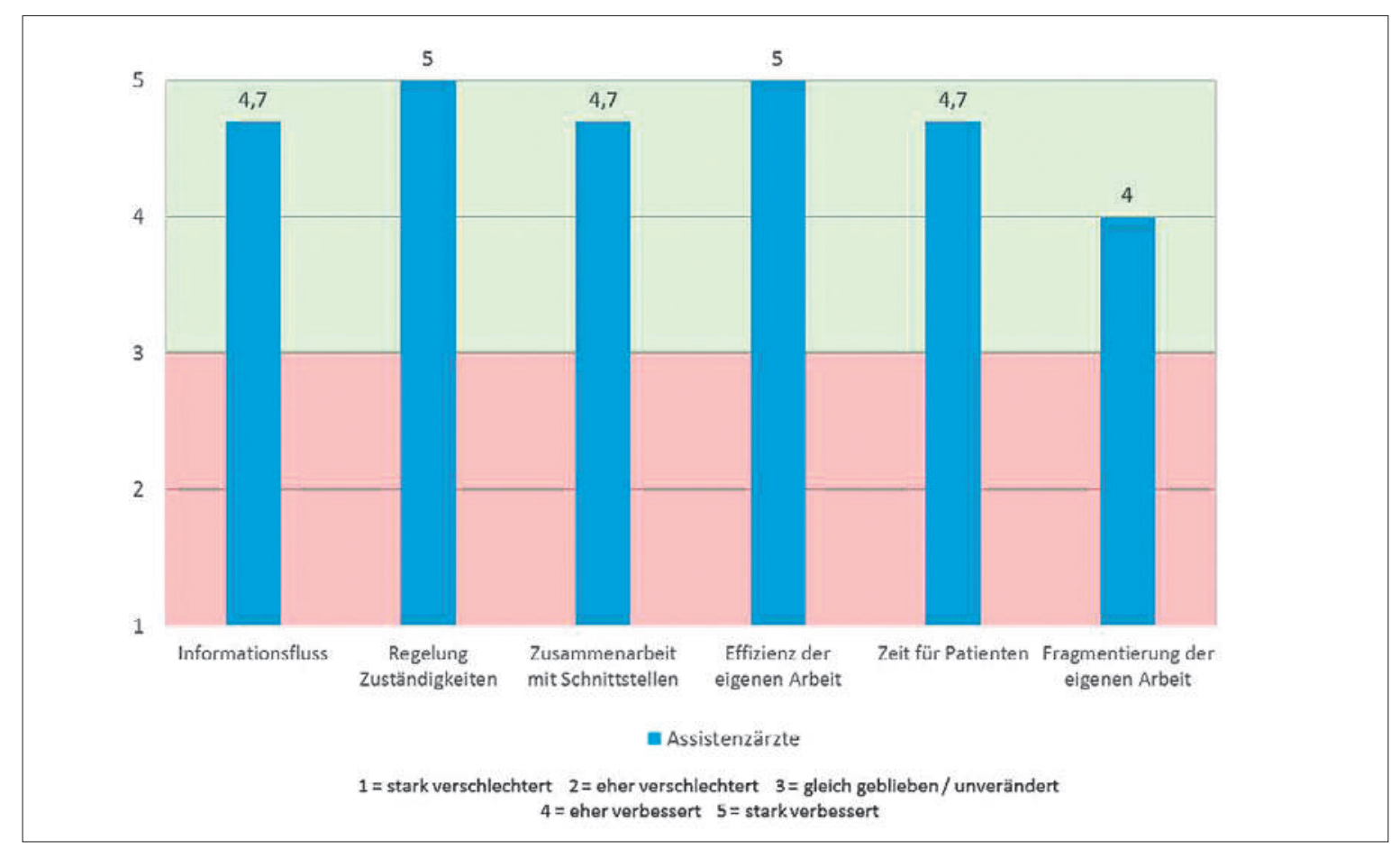

Abbildung 2: Einschätzung der Veränderungen durch das Hospitalisationsmanagement (Grafik: Auszug, modifiziert nach Schade $\mathrm{V}$ et al. [10]). 
Verbesserungen, die durch das Hospitalisationsmanagement erzielt wurden (siehe Abbildung 2). Zusätzlich schätzten die Ärzte die besser verfügbaren und aktualisierten Krankengeschichten sehr.

Auch andere Berufsgruppen profitieren vom Hospitalisationsmanagement. Pflege und Ambulante Dienste erledigten weniger administrative Tätigkeiten für die Ärzte (z.B. Formularwesen, Vereinbarung von Konsilien und Untersuchungen, Kopierarbeiten etc.). Das Hospitalisationsmanagement trug zu einer besseren Zusammenarbeit innerhalb der Kliniken und mit externen Schnittstellen bei.

In mehreren Projektevaluationen zeigte sich, dass sich durch die Einführung des Hospitalisationsmanagements in den betreffenden Kliniken bzw. Spitälern die Arbeitssituation auf breiter Ebene verbessert hat. Neben den angezielten Verbesserungen konnte auch die Arbeitszufriedenheit der Ärzte erhöht werden. Angesichts der grossen Herausforderungen, die ärztliche Tätigkeit mit sich bringt, ist das nicht zu vernachlässigen. Und: Mit dem Hospitalisationsmanagement werden attraktive Stellen geschaffen. Stellen, die wesentlich einfacher zu besetzen sind als ärztliche.

Vereinfacht könnte man es so ausdrücken: Die Aufgabenverteilung in den Kliniken um den Patienten herum wird wieder zurechtgerückt.

\section{Zusammenfassend}

Rundum Zufriedenheit also? Für die Ärzte und weitere beteiligte Berufsgruppen, das zeigen unsere Erfahrungen, lässt sich das bejahen. Aber: Das Hospitalisationsmanagement kostet Geld. Geld, welches sichtbar die Budgets belastet. Allerdings lassen die oben beschriebenen Verbesserungen erwarten, dass dadurch vielmehr Kosten gespart werden können.

Das Bemühen um die administrative Entlastung der Ärzte und eine sinnvolle Aufgabenverteilung im Spital hat verschiedene Gesichter. Mancherorts sind Aufgabenerweiterungen der Stationssekretariate oder die Übertragung von Aufgaben an die Pflege zu beobachten. Allerdings ist es nicht unproblematisch, wenn man diesen Berufsgruppen zusätzliche Aufgaben überträgt, da deren Arbeitsbelastung ebenfalls hoch ist. Zudem fehlt es häufig an der nötigen konzeptuellen Grundlage solcher Aufgabenverschiebungen.

Die Ausbildung der Sekretariate ist ausreichend für den administrativen Teil der Aufgaben des Hospitalisationsmanagements, jene der Pflege für den medizinischen. Gebraucht wird die Kombination von beidem. Es braucht also eine klar konzipierte neue Funktion. Das Hospitalisationsmanagement greift die bestehenden Probleme auf und fügt sie in ein erprobtes Konzept ein.

«Die Hospitalisationsmanagerin schenkt uns Zeit für andere Dinge, wie die Untersuchung von Patienten und Gespräche mit Angehörigen» (Aussage eines Arztes).

Dem ist eigentlich nichts hinzuzufügen.

\section{Literatur}

1 VSAO. Arbeitsbelastung der Assistenz- und Oberärztinnen und -ärzte. Mitgliederbefragung, 2014. www2.vsao.ch/fileupload/ 201449144848_pdf.pdf

2 VSAO. Arbeitsbelastung der Assistenz- und Oberärztinnen und -ärzte. Management Summary, 2014. www2.vsao.ch/fileupload/ 201441483759_pdf.pdf

3 VSAO. Illegale Arbeitsbedingungen für Assistenz- und Oberärzte Medienmitteilung, 2014. www2.vsao.ch/fileupload/201441483124 _pdf.pdf

4 Meyer B, Rohner B, Golder L, Longchamp C. Administrativer Aufwand für Ärzte steigt weiter an: Schweiz Ärztezeitung. 2016;97(1):6-8.

5 Hehli S. Zu viel Bürokratie: Mediziner versinken in Papierflut. NZZ, 2016. www.nzz.ch/schweiz/mediziner-versinken-in-der-papier flut-1.18672428

6 Golder L, Longchamp C, Jans C, Tschöpe S, Venetz A, Hagemann M, Schwab J. Trotz steigendem Dossieraufwand bleibt die Spitalärzteschaft motiviert: Begleitstudie anlässlich der Einführung von SwissDRG sowie der geplanten stationären Tarife in der Rehabilitation und Psychiatrie im Auftrag der FMH, 5. Befragung. Gfs.bern, 2015. www.fmh.ch/files/pdf17/20160106_FMH-Begleitforschung Schlussbericht_D.pdf

7 Schoch C. Assistenz- und Oberärzte arbeiten zu lange: Gefahren für die Sicherheit der Patienten. NZZ, 2014. www.nzz.ch/schweiz/ gefahren-fuer-die-sicherheit-der-patienten-1.18283852

8 Siegrist M, Gutscher M, Giger M. Was haben Betriebskultur, Motivation und Fremdjahr mit der Weiterbildung zu tun? Die wichtigsten Ergebnisse der Assistentenumfrage 2003: Schweiz Ärztezeitung. 2004;85(15):783-91.

9 Semmer NK, Jacobshagen N, Meier LL, Elfering A, Kälin W et al. Psychische Beanspruchung durch illegitime Aufgaben. Immer schneller, immer mehr. Springer Fachmedien Wiesbaden, 2013. 97-112.

10 Schade V, Graf N, Bühler R, Sturzenegger M. Case-Management in der Neurologie: Entlastung durch Verlagerung: Inselbote. 2003;4:37-41. 